\title{
Joint modelling of time-to-clinical malaria and parasite count in a cohort in an endemic area
}

Christopher C. Stanley 1,2 ${ }^{*}$, Lawrence N. Kazembe ${ }^{3}$, Andrea G. Buchwald ${ }^{4}$, Mavuto Mukaka5,6, Don P. Mathanga ${ }^{2}$, Michael G. Hudgens ${ }^{7}$, Miriam K. Laufer ${ }^{4}$ and Tobias F. Chirwa ${ }^{1}$

*Correspondence: stanleychristopher1@yahoo.com

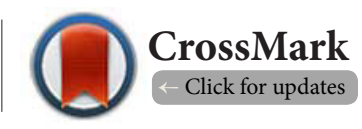

1School of Public Health, Faculty of Health Sciences, University of the Witwatersrand, Johannesburg, South Africa.
2Malaria Alert Center, University of Malawi College of Medicine, Blantyre, Malawi.
${ }^{3}$ Department of Statistics, University of Namibia, Windhoek, Namibia.
${ }^{4}$ Center for Vaccine Development and Global Health, University of Maryland School of Medicine, Baltimore, USA.
${ }^{5}$ Oxford Centre for Tropical Medicine and Global Health, Oxford, United Kingdom.
${ }^{6}$ Mahidol-Oxford Tropical Medicine Research Unit, Bangkok, Thailand.
${ }^{7}$ Department of Biostatistics, Center for AIDS Research (CFAR), University of North Carolina Chapel Hill, North Carolina, USA.

\begin{abstract}
Background: In malaria endemic areas such as sub-Saharan Africa, repeated exposure to malaria results in acquired immunity to clinical disease but not infection. In prospective studies, time-to-clinical malaria and longitudinal parasite count trajectory are often analysed separately which may result in inefficient estimates since these two processes can be associated. Including parasite count as a time-dependent covariate in a model of time-to-clinical malaria episode may also be inaccurate because while clinical malaria disease frequently leads to treatment which may instantly affect the level of parasite count, standard time-to-event models require that time-dependent covariates be external to the event process. We investigated whether jointly modelling time-to-clinical malaria disease and longitudinal parasite count improves precision in risk factor estimates and assessed the strength of association between the hazard of clinical malaria and parasite count.
\end{abstract}

Methods: Using a cohort data of participants enrolled with uncomplicated malaria in Malawi, a conventional Cox Proportional Hazards (PH) model of time-to-first clinical malaria episode with time-dependent parasite count was compared with three competing joint models. The joint models had different association structures linking a quasi-Poisson mixed-effects of parasite count and event-time Cox PH sub-models.

Results: There were 120 participants of whom 115 (95.8\%) had $>1$ follow-up visit and $100(87.5 \%)$ experienced the episode. Adults >15 years being reference, $\log$ hazard ratio for children $<5$ years was 0.74 (95\% CI: $0.17,1.26)$ in the joint model with best fit vs. 0.62 (95\% CI: 0.04, 1.18) from the conventional Cox PH model. The log hazard ratio for the 5-15 years was 0.72 (95\% CI: 0.22, 1.22) in the joint model vs.0.63 (95\% CI: $0.11,1.17)$ in the Cox PH model. The area under parasite count trajectory was strongly associated with the risk of clinical malaria, with a unit increase corresponding to -0.0012 (95\% CI: -0.0021, -0.0004) decrease in log hazard ratio.

Conclusion: Jointly modelling longitudinal parasite count and time-to-clinical malaria disease improves precision in log hazard ratio estimates compared to conventional time-dependent Cox PH model. The improved precision of joint modelling may improve study efficiency and allow for design of clinical trials with relatively lower sample sizes with increased power.

Keywords: Prospective studies, longitudinal data, malaria parasite, time-to-event, clinical malaria, Cox proportional hazards model, joint modelling

(c) 2019 Stanley et al; licensee Herbert Publications Ltd. This is an Open Access article distributed under the terms of Creative Commons Attribution License (http://creativecommons.org/licenses/by/3.0). This permits unrestricted use, distribution, and reproduction in any medium, provided the original work is properly cited. 
Stanley et al., Journal of Medical Statistics and Informatics 2019,

\section{Introduction}

Malaria remains one of the most common parasitic infections globally with a disproportionately high burden in sub-Saharan Africa [1]. In malaria-endemic areas, repeated exposure results in acquired immunity to clinical malaria disease but not infection. Of interest in many malaria studies is to estimate time-to-clinical malaria but repeated exposure may give rise to a relationship between the disease and time-dependent covariates such as parasite count. However, time-to-clinical malaria and parasite count data are often analysed separately, mostly using Cox proportional hazards (PH) models and mixed-effects models or generalised estimating equations (GEE) respectively [2-4]. Separate analysis of time-to-clinical malaria disease and parasite count data may result in inefficient estimates when these two processes are strongly associated [5].

For accurate estimation of the risk of clinical malaria, analytical methods are required that account for historical exposure and the relationship between clinical malaria and infection parasite count. To investigate the strength of this association, one approach would be including the parasite countas a timedependent covariate in the model of time-to-clinical malaria episode. However, this approach may be inaccurate because it does not account for the fact that parasite count in this case is an endogenous covariate whose existence and future path can be directly related to the occurrence of the episodes [6]. Standard time-to-event models require that time-dependent covariates be external to the event process [7] but clinical malaria disease frequently leads to treatment which may instantly affect the level of parasite count.

A second approach is to fit a joint model of time-to-clinical malaria and longitudinal parasite count profile. Previous applications of the joint modelling framework are many. These include, for example, analysis of CD4 count jointly with timeto-development of AIDS [8-12] and modelling quality of life performance scores jointly with time-to-death or disease progression among patients with cancer $[11,13,14]$. In order to fit joint models to data from malaria studies, there are certain aspects of these types of data that require consideration. In particular, following treatment it is possible for the parasite count to equal zero, so the joint model should allow for that.

We investigated whether modelling time-to-new clinical malaria disease jointly with parasite count trajectory data may improve precision in log hazard ratio estimates when compared to the conventional Cox $\mathrm{PH}$ model with timedependent parasite count. We also assessed the strength of the association between the hazard of clinical malaria and a time-varying parasite count.

\section{Methodology \\ Data source}

The study was motivated by data from the Mfera prospective cohort study conducted in Chikwawa district, southern Malawi, described previously by Buchwald and others [15]. Malaria disease is endemic in Malawi [16] and transmission of the Plasmodium falciparum parasite is high in Chikwawa $[17,18]$. The cohort enrolled 120 participants who presented with uncomplicated malaria at the Mfera health centre in the Chikwawa district between June 2014 and March 2015. Initial diagnosis was made by rapid diagnostic test (RDT) and confirmed by microscopy using thick blood smears. Exclusion criteria from the Mfera cohort included: acute illness requiring hospitalization, signs or symptoms of severe malaria or moderate to severe anemia, and chronic medication with any drug that has antimalarial activity e.g. HIV treatment. Participants underwent passive and active surveillance on a monthly basis and whenever sick for up to two years to assess re-infection, host response and parasite count.

\section{Primary outcome}

In the current analyses, the outcome of interest was time-to-first new clinical malaria disease which was defined by participants' self-reported fever and apositive RDT result.

\section{Notation and specification of the models}

The conventional Cox PH model with time-dependent parasite count is defined as in [9] with the hazard function $\lambda_{i}(t)$ for participant at given time expressed as.

$$
\lambda_{i}(t)=\lambda_{0}(t) \exp \left[\beta_{s} X_{s i}^{\prime}(t)\right]
$$

where and $\lambda_{0}(t)$ is the unspecified baseline hazard function and the covariate vector $X_{s i}(t)$ for participant includes participant's age and frequency of insecticide treated bed nets use in the previous month of the visit. Covariates were included in multivariable models if they were significant at alpha level of 0.1 in univariate $\mathrm{Cox} \mathrm{PH}$ models.

For the joint models, we utilised the Bayesian joint modelling approach for longitudinal and survival data proposed by Chen et al [12] which fits a model with Markov Chain Monte Carlo (MCMC) methods as presented by Ibrahim et al [19]. The joint model is composed of longitudinal and survival sub-models. The longitudinal sub-model takes the form of a mixed-effects model as follows; supposing data is available from $N$ participants with $n_{i}$ observations recorded for participant $i,(i=1, \ldots \ldots, N)$. The response $y_{i j}\left(j=1, \ldots \ldots . ., n_{i}\right)$, fixedeffect covariate vector $X_{i j}=\left(X_{1 i j}, \ldots . . . ., X_{p i j}\right)^{\prime}$, and random-effect covariate vector $Z_{i j}=\left(Z_{1 i j}, \ldots . . . ., Z_{q i j}\right)^{\prime}$ are recorded at times $t_{i j}$. The longitudinal sub-model is

$$
y_{i j}=\beta X_{i j}^{\prime}+b_{i} Z_{i j}^{\prime}+\varepsilon_{i j}
$$

where $\beta$ is the $p \times 1$ fixed-effect parameter vector, $b_{i}$ is the $q \times 1$ vector of random effects for participant which is assumed to be multivariate normal with mean zero, i.e., $b \sim N_{q}\left(0, \Sigma_{b}\right)$, and $\Sigma_{b}$ is the variance-covariance matrix of the subject specific effects. The error vector $\varepsilon_{i}=\left(\varepsilon_{i 1}, . ., \varepsilon_{n j}{ }^{i}\right)^{\prime}$ is assumed to be distributed $\varepsilon_{i} \sim N_{n}{ }^{i}$ $\left(0, \delta^{2} I_{n i}\right)$ where $\delta^{2}$ is variance and $I_{n i}$ is the $n_{i} \times n_{i}$ identity matrix.

The survival sub-model takes a Cox PH model form [9] where 
the hazard function for $\lambda_{i}(t)$ participant $i$ at time $t$ as given in equation (1) is modelled as

$$
\lambda_{i}(t)=\lambda_{0}(t) \exp \left[\theta h\left(\beta, b_{i}, t\right)+\beta_{s} X_{s i}^{\prime}(t)\right],
$$

where $h(\beta, b, t)$ is a function of the fixed and random effects in the longitudinal sub-model, and $\theta$ is an association parameter linking the two sub-models: survival and longitudinal models. The $\beta_{s}$ is a parameter vector for covariates unique to the survival sub-model. The survival covariate vector $X_{s i}(t)=\left(x_{s i l} \ldots, X_{\text {sil }}\right)^{\prime}$ may include baseline covariates for participant $i$ with $\beta_{s}$ representing $r \times 1$ parameter vector. The functional form of $h(\beta, b, t)$ determines the type of the association structure between longitudinal parasite count and the time-to new clinical malaria episode. Taking the first derivative of $h(\beta, b, t)$ is interpreted in terms of an association of the rate of change in parasite count at time and the hazard of new clinical malaria episode at the same time, while the integral of $h(\beta, b, t)$ would relate the hazard and the cumulative parasite count trajectory defined as area under parasite count profile from baseline up to the time of the new episode.

Once the two sub-models are specified, the likelihood for the joint model can be constructed as follows. Let $T_{i}$ and $C$ represent potential failure and censoring times respectively for participant $i$. Let $S_{i}=\min \left\{T_{p} C_{j}\right\}$ be the minimum of the observed failure and censoring times for participant $i$, and let $\tau_{i}$ be the failure indicator taking value 1 if $T_{i} \leq C_{i}$ and 0 otherwise. Then the value of the longitudinal trajectory for participant $i$ at time $t$ can be defined as $\varphi_{i}(\beta, b, t)$ and at visit $j$ as $\varphi_{i j}(\beta, b)$. Using the full longitudinal trajectory, then the likelihood of the joint distribution of the observed data and random effects for participant can be decomposed as

$$
\begin{aligned}
& L_{i} \propto f_{i}(\text { Survival } \mid \text { longitudinal }) \times f_{i}(\text { longitudinal }) \\
& =f\left(S_{i} \mid \theta, \tau_{i}, \beta_{s}, \varphi_{i}\left(\beta, b_{i}, t\right), X_{s i}\right) \times f\left(y_{i} \mid x_{i}, z_{i}, \beta, b_{i}\right) f\left(b_{i}\right)
\end{aligned}
$$

and the joint likelihood for all participants can be written as $L=\prod_{i=1}^{N} L_{i=1}$.

Denoting parasite count as $\mathrm{PC}$, the likelihood of the joint distribution of observed data, random effects and PC can be expressed as

$$
L=f\left(S \mid \theta, \tau, \beta_{s}, \varphi(\beta, b, t, P C)\right) \times f(b, P C \mid \beta)
$$

and integrating out the random effects of the conditional likelihood yields the marginal likelihood. Under the Bayesian framework, the random effects are sampled in MCMC algorithm as extra parameters. The survival and longitudinal sub-models are linked by sharing common random effects structure. The MCMC computations of the model parameters proceed assuming that given the random effects, the longitudinal parasite countand time-to-clinical malaria process are independent as are the longitudinal responses of each participant.

\section{Data analysis}

Baseline data was summarised using frequencies and percentages if categorical and medians with ranges were presented if continuous and skewed. Failure functions for time to new clinical malaria disease were summarised using Kaplan Meier plots, and compared across age and bed net usage using logrank test. Four models of time-to-new clinical malaria disease were fitted. These included the reference model (model 1), a conventional $\mathrm{Cox} \mathrm{PH}$ model fitted with time-dependent parasite count measured at each visit, and three competing joint models. The three joint models take a common formulation only differing in the assumed association structure linking the hazard of clinical malaria with parasite count. The hazard of clinical malaria disease at any time was linked with: 1) the current underlying value of parasite count at the same time point (model 2), 2) the rate of change in parasite count trajectory at time (model 3 ), and 3 ) the cumulative trajectory or area under profile of parasite count from baseline up to time (model 4). Each joint model involved fitting a quasi-Poisson mixed-effects sub-model of parasite count longitudinal trajectory with natural spline functions of time and including the resulting estimates as covariates in the Cox $\mathrm{PH}$ sub-model. Other covariates in the conventional $\mathrm{Cox} \mathrm{PH}$ and survival sub-models included age of the participant and frequency of insecticide treated bed net use in previous month. Parameters were estimated using MCMC through the random walk Metropolis-Hastings (M-H) algorithm. Diffused normal priors were assumed for the covariates including the association parameter. Diagnostic assessments were conducted to assess the convergence of the MCMC samples using trace and kernel density estimator plots, for the final optimal model. Analyses were done in Stata SE version 15.1 (Stata Corp., College Station, TX) [20] using programme bayesmh and $\mathrm{R}$ version 3.4.3 using packages JMbayes, survival and glmmPQL [21].

\section{Results}

There were 120 participants in the cohort, of which 69 (57.5\%) were females. The overall median age was7.5 years [inter-quartile range (IQR): 4.7-18.1], 6.3 years (IQR: 3.2-13.1) for males and 9.2 years (IQR: 5.3-18.5) females (Table 1). The median number of malarial parasites per $\mu \mathrm{L}$ was 11,060 (IQR: 840-54,000) overall, 24,840 (IQR: 1,600-68,600) in males, and 5,640 (IQR: 520-540, 000 ) for females. During enrolment, 48 (44.9\%) out of 107 particiants reported to have been using bed nets every night in previous month.

Follow up and time-to-first new clinical malaria disease Analyses of time-to-new clinical malaria disease included 115 participants who had a least one follow-up visit post enrolment and together contributed a total 894 observations. The median follow-up time to new clinical malaria disease episode was 3.5 months (IQR: 1.1-7.9). Out of the 115 participants, 100 (87.5\%) experienced the episode while 15 (12.5\%) were administratively right censored (Table 2). Among 100 participants 
Stanley et al., Journal of Medical Statistics and Informatics 2019,

http://www.hoajonline.com/journals/pdf/2053-7662-7-1.pdf

doi: $10.7243 / 2053-7662-7-1$

Table 1. Baseline demographic, clinical conditions and vital signs for Mfera malaria cohort in Malawi.

\begin{tabular}{|c|c|}
\hline Variable & Total $(n=120)$ \\
\hline Gender, female, n (\%) & $69(57.4)$ \\
\hline \multicolumn{2}{|l|}{ Age, n (\%) } \\
\hline$<5$ years & $34(28.3)$ \\
\hline $5-15$ years & $51(42.5)$ \\
\hline$>15$ years & $35(29.2)$ \\
\hline Weight $(\mathrm{kg})$, median (IQR) & $21.5(15.0-46.0)$ \\
\hline Height $(\mathrm{cm})$, median $(\mathrm{IQR})$ & $119.5(103.0-151.8)$ \\
\hline Temperature $\left({ }^{\circ} \mathrm{C}\right)$, median (IQR) & $36.7(36.2-38.6)$ \\
\hline $\begin{array}{l}\text { Respiratory rate (breaths/minute), median } \\
\text { (IQR) }\end{array}$ & $28(22-36)$ \\
\hline Heart rate (beats/minute), median (IQR) & $112(92-139)$ \\
\hline Haemoglobin (g/dl), median (IQR) & $11.5(10.2-12.4)$ \\
\hline $\begin{array}{l}\text { Parasite count (number of parasites } / \mu \mathrm{L} \text { ), } \\
\text { median (IQR) }\end{array}$ & $11060(840-54000)$ \\
\hline Cough, n (\%) & $15(12.5)$ \\
\hline Musculoskeletal pain, n (\%) & $40(33.3)$ \\
\hline Headache, n (\%) & $36(30.0)$ \\
\hline Vomiting, n (\%) & $32(26.7)$ \\
\hline Abdominal pain, $\mathrm{n}(\%)$ & $15(12.5)$ \\
\hline \multicolumn{2}{|l|}{ Bed net use previous month*, n(\%) } \\
\hline Every night & $48(44.9)$ \\
\hline Most nights ( $>$ half) & $13(12.1)$ \\
\hline Some nights $(<$ half $)$ & $8(7.5)$ \\
\hline No nights & $38(35.5)$ \\
\hline \multicolumn{2}{|l|}{ Season enrolled, $\mathbf{n}(\%)$} \\
\hline Dry: May - November & $91(75.8)$ \\
\hline Rainy: December - April & $29(24.2)$ \\
\hline
\end{tabular}

${ }^{*}$ not adding up to column total due to missing

Table 2. Characteristics by clinical malaria statusfor Mfera malaria cohort in Malawi.

\begin{tabular}{lll}
\hline Variable & $\begin{array}{l}\text { Clinical malaria } \\
\text { episode }(\mathbf{n}=\mathbf{1 0 0})\end{array}$ & $\begin{array}{l}\text { No clinical malaria } \\
\text { episode }(\mathbf{n}=\mathbf{1 5})\end{array}$ \\
\hline Sex, $\mathbf{n}(\%)$ & & \\
\hline Male & $42(42.0)$ & $8(53.3)$ \\
Female & $58(58.0)$ & $7(47.7)$ \\
Age, $\mathbf{n}(\%)$ & & $4(26.7)$ \\
$<5$ years & $27(27.0)$ & $2(13.3)$ \\
$5-15$ years & $48(48.0)$ & $9(60.0)$ \\
$>15$ years & $25(25.0)$ & $8(53.3)$ \\
\hline Net use previous month, n(\%) & $7(46.7)$ \\
\hline $\begin{array}{l}\text { Every night } \\
\text { Not every night }\end{array}$ & $37(37.0)$ & $12.3(10.9-13.9)$ \\
$\begin{array}{l}\text { Haemoglobin (g/dl) } \\
\text { Parasite count, }\end{array}$ & $11.4(10.0-12.3)$ & \\
number of parasites & $13640(840-52040)$ & $2800(560-60040)$ \\
per $\mu \mathrm{L}$, median (IQR) & & \\
\hline
\end{tabular}

Note: clinical malaria status data available for 115 participants who had a least one follow-up visit. who experienced the episode, 58 (58\%) were females, 48 (48\%) were aged 5-15 years, 37 (37\%) reported using bed net nightly in prior month to enrolment and their median parasite count was 13640 (IQR: 840 - 52040).

\section{Parameter estimation}

Regression coefficient estimates are log hazard ratios, log (HRs). Overall, the joint models gave larger log (HR) estimates with consistently smaller standard errors and narrower credible intervals when compared to the conventional Cox PH model with time-dependent parasite count (Table 3). Comparing the Deviance Information Criteria (DIC) from the three joint models showed that joint model 4 with cumulative parasite count had the lowest value (Table 4), suggesting the best fit [22]. For the rest of the manuscript, the joint model is referencing to the joint model 4 with cumulative parasite count which is being compared to the conventional Cox PH model (model 1). Considering adults above 15 years as reference group, the $\log (\mathrm{HR})$ of clinical malaria disease for children under 5 years, was 0.74 [95\% Credible Interval (Cl): $0.17,1.26$ ] in the joint model compared to $0.62(95 \% \mathrm{Cl}: 0.04,1.18)$ from the conventional Cox PH model. The log (HR) for participants aged $5-15$ years was $0.72(95 \% \mathrm{Cl}: 0.22,1.22)$ in the joint model compared to $0.63(95 \% \mathrm{Cl}: 0.11,1.17)$ in the conventional Cox PH model. Considering participants who used a bed net nightly in the previous month as reference, the log (HR) of clinical malaria disease for those who did not use a bed net every night was 0.58 (95\% Cl: $0.13,1.07)$ from the joint model compared to 0.52 (95\% Cl: $0.07,1.08)$ in the conventional Cox PH model. From the joint model, the area under the longitudinal trajectory of parasite count was strongly associated with the risk of clinical malaria, with a unit increase corresponding to a -0.0012 (95\% Cl: $-0.0021,-0.0004)$ decrease in the log hazard ratio. Neither current underlying value of parasite count nor rate of change in parasite count trajectory at the same time point were significantly associated with the occurrence of a new clinical malaria episode.

\section{MCMC convergence of estimated log (HR) parameters from final optimal joint model}

The MCMC sampler trace plots from the final joint model 4 seemed to mix well for parameters of children under 5 years, 5-15 years and infrequent bed net use and never moved beyond 1.5 achieving convergence (Figure $\mathbf{2 A - 2 C}$ ). The sampler for cumulative parasite count parameter, showed noisy pattern before converging at about 1000 iterations (Figure 2D). From the Kernel density estimator plots, all the four parameters were roughly normal suggesting that the $\mathrm{M}-\mathrm{H}$ algorithm sampled within the target normal distribution (Figure 3A-3D).

Factors associated with risk of new clinical malaria episode In unadjusted analyses using Kaplan Meier failure estimator, the risk of experiencing new clinical malaria episode was higher in children under 5 years and the 5-15 years compared 
Table 3. Log of hazard ratio estimates for time-to-new clinical malaria episode for Mfera cohort in Malawi.

\begin{tabular}{llllll}
\hline & \multicolumn{2}{l}{ Age at baseline $^{*}$} & $\begin{array}{l}\text { Bed net use in } \\
\text { previous month }\end{array}$ & Parasite count $^{+}$ \\
\hline Model & Parameter & $\theta_{<5 \text { years }}$ & $\theta_{5-15 \text { years }}$ & $\theta_{\text {not every night }}$ & $\theta_{\text {parasite count }}$ \\
\hline Model 1 & Estimate & 0.624 & 0.632 & 0.520 & $-1.77 \mathrm{e}-5$ \\
& Std error & 0.282 & 0.259 & 0.292 & $1.86 \mathrm{e}-6$ \\
& $95 \%$ CI & $0.044,1.183$ & $0.115,1.174$ & $0.076,1.085$ & $-1.41 \mathrm{e}-5,2.14 \mathrm{e}-5$ \\
\hline Model 2 & Estimate & 0.672 & 0.707 & 0.582 & -0.029 \\
& Std error & 0.016 & 0.014 & 0.024 & 0.053 \\
& $95 \%$ CI & $0.094,1.251$ & $0.213,1.265$ & $0.110,1.017$ & $-0.345,0.411$ \\
\hline Model 3 & Estimate & 0.749 & 0.775 & 0.594 & -0.103 \\
& Std error & 0.023 & 0.021 & 0.023 & 0.071 \\
& $95 \%$ CI & $0.077,1.485$ & $0.193,1.253$ & $0.159,1.156$ & $-6.168,6.124$ \\
\hline Model 4 & Estimate & 0.735 & 0.722 & 0.575 & -0.001 \\
& Std error & 0.022 & 0.028 & 0.030 & 0.0001 \\
& $95 \%$ CI & $0.170,1.263$ & $0.219,1.215$ & $0.125,1.066$ & $-0.0021,-0.0004$ \\
\hline
\end{tabular}

Log hazard ratio estimates are posterior means. All models are multivariable. $\mathrm{CI}=$ Credible Interval. ${ }^{\star}$ reference: $>15$ years, +reference: bed net use nightly.

Table 4. Deviance Information Criteria (DIC) from the competing joint models.

\begin{tabular}{ll}
\hline Joint Model & DIC \\
\hline Model 2: Joint model with current underlying value of parasite count at any time & 74763360 \\
Model 3: Joint model with rate of change in parasite count trajectory at any time & 74763436 \\
Model 4: Joint model with cumulative parasite count from baseline up to any time & 74763318 \\
\hline
\end{tabular}

to adults above 15 years (log-rank p-value=0.016) (Figure 1A). The risk of getting a new clinical malaria episode was also higher in participants who did not use bed net every night compared to those who used a bed net nightly in previous month (log-rank p-value=0.016) (Figure 1B). Based on the joint model, conditional on cumulative parasite count profile, the hazard of getting new clinical malaria episode was higher in children under 5 years by 2.1 -fold $(95 \% \mathrm{Cl}: 1.2,3.4)$ and those aged $5-15$ years by 2.0 -fold $(95 \% \mathrm{Cl}: 1.2,3.5)$ when compared to adults over 15 years old and controlling for bed net usage. Among participants of the same age, the conditional hazard was also higher for participants who did not use a bed net every night by 1.8 -fold $(95 \% \mathrm{Cl}: 1.1,3.3)$ compared to those who used the bed net nightly in previous month.

\section{Discussion}

This study has demonstrated that jointly modelling longitudinal parasite count and time-to clinical malaria episodes improves precision in risk factor estimates associated with clinical malaria disease. The joint model yielded larger parameter estimates with consistently smaller standard errors and narrower credible intervals when compared to a conventional Cox PH model with time-dependent parasite count. These results are consistent with findings from other areas including HIV $[8,12]$ and cancer $[13,14,23]$ where joint modelling out-performs separate analyses in terms of optimal use of the available information giving both more precise and less biased estimates. The improved precision provided by the joint model may improve study efficiency, for example, clinical trials may be designed with relatively lower sample sizes while still yielding high power. In general, the conventional time-dependent Cox PH model like any other standard timeto-event model assume that time-dependent covariates are external to the event process [7]. However, parasite count in this case is an endogenous covariate whose existence and future path can be directly related to the occurrence of the malaria episodes. By postulating a model for the joint distribution of the covariate parasite count and the time-to-malaria processes, the joint model explicitly accounts for possible inter-dependence between the two processes through shared random effects [24]. Moreover, the time-dependent covariate model assumes that the value of the parasite count does not change until a new measurement is taken which may not be correct. When we modelled parasite count using a quasiPoisson mixed effects model, we were creating a model for the outcome at any time-point there by indirectly addressing the measurement error. The joint model also offered flexibility to investigate the appropriate link structure between longitudinal parasite count and time-to-new clinical malaria episode. Among the fitted joint models, only the model with cumulative parasite count was strongly associated with the hazard of new clinical malaria episode suggesting that the risk can 

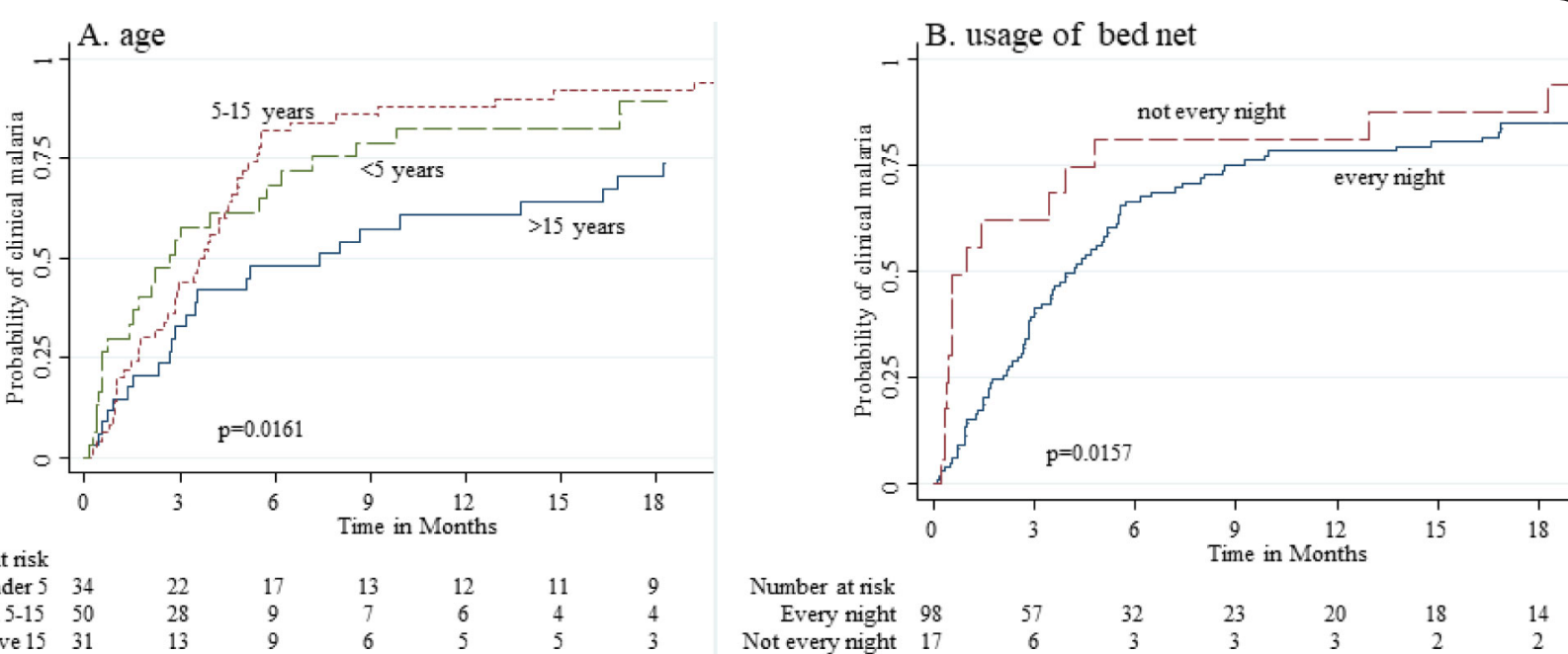

Number at risk

Children under $5 \quad 34$

Children aged $5-15 \quad 50$

Adults above $15 \quad 31$

$\begin{array}{llllll}22 & 17 & 13 & 12 & 11 & 9 \\ 28 & 9 & 7 & 6 & 4 & 4 \\ 13 & 9 & 6 & 5 & 5 & 3\end{array}$

Figure 1. Kaplan-Meier estimator for time-to-new clinical malaria episode by A) baseline age, and B) use of bed net in previous month. The risk of experiencing clinical malaria episode was significantly higher in children under 5 years and of 5-15 years, and among participants who did not use bed nets every night.

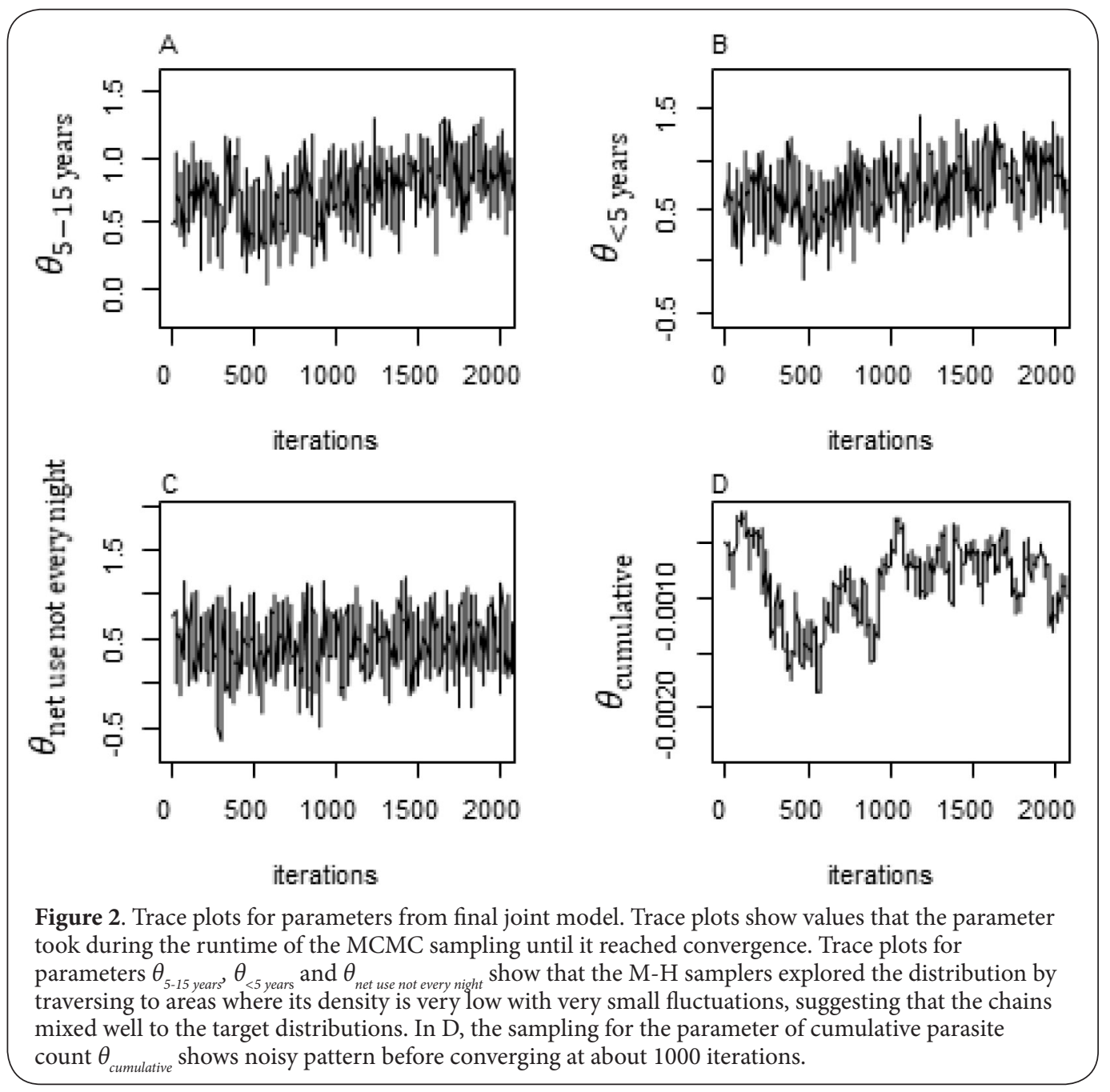




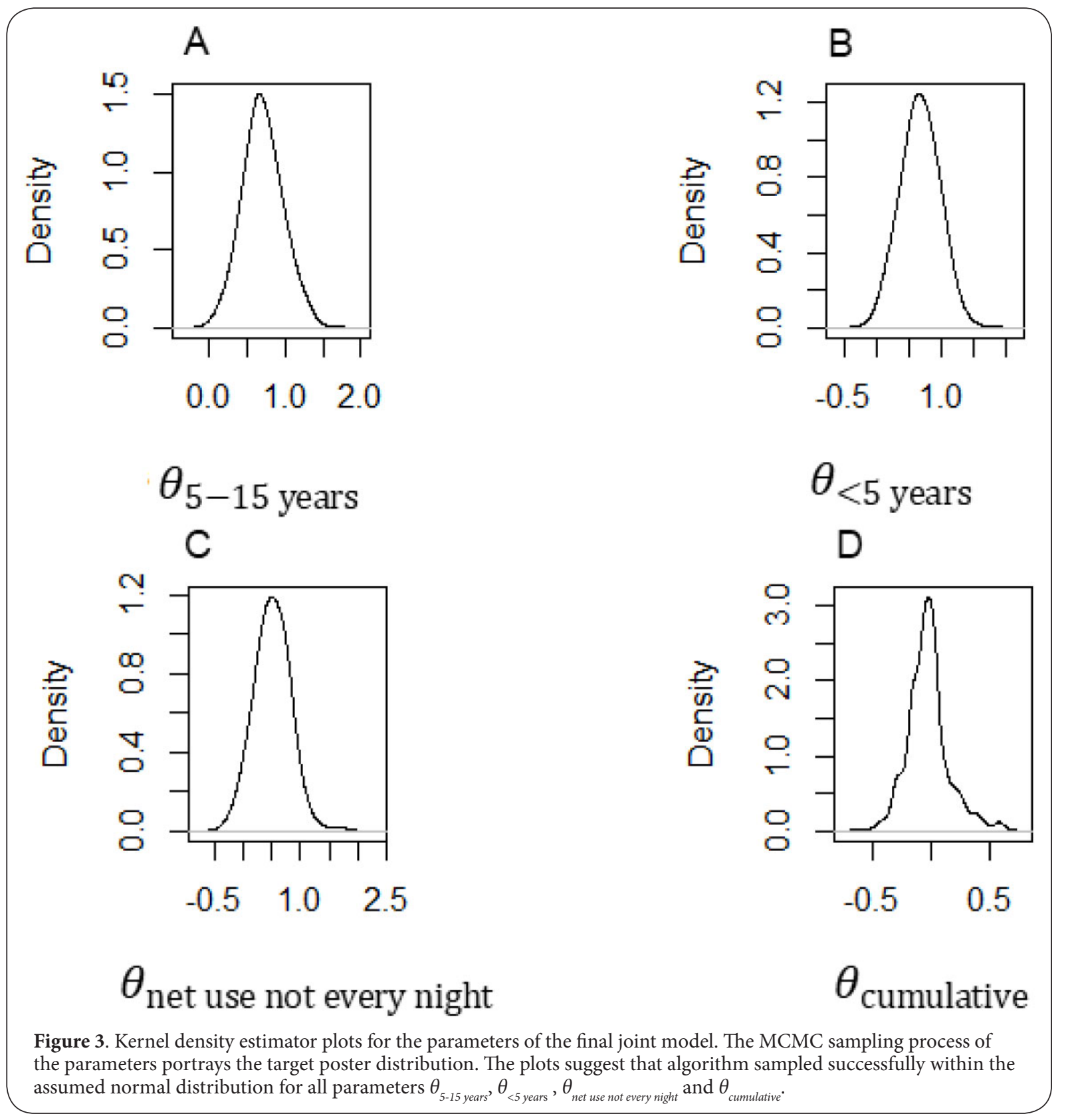

better be explained by conditioning on the cumulative effect of the longitudinal parasite count trajectory from baseline up to the episode time. The underlying current value of the parasite count or change in parasite count at any time was not associated with the risk of experiencing new clinical malaria episode at the same.

Factors associated with a high risk of experiencing a new clinical malaria episode were young age and infrequent use of bed nets as have been reported in other studies [25-28]. Children aged up to 15 years had higher risk for clinical malaria disease when compared to adults over 15 years. The higher risk of experiencing a clinical malaria episode among children is possibly be due to the naturally under-developed humoral immune responses to different stage-specific antigens of $P$. Falciparum otherwise acquired with age [29]. In this study in an endemic area, high cumulative parasite count was associated with lower risk of getting a new clinical malaria episode suggesting that increased exposure to malaria parasites with time may result into protective effect to future clinical malaria episodes.

This study may be limited by focusing on time-to-first malaria episode only, thus estimates obtained here may not be applicable to analyses examining all clinical malaria episodes over a follow up period. This paper has established the optimal 
way of incorporating parasite count in estimating time-to-first new clinical malaria which may further be extended to study recurrent episodes over entire follow-up, but this may require different distributional assumptions. There were missing values for bed net use which may have affected the findings. Future studies should include multiple imputation of missing covariate data and also investigate the role of measurement error due to detection limit of parasite count in these joint models. Further work is required to consider joint modelling of parasite count with recurrent episodes and to predict risk of future clinical malaria episodes.

\section{Conclusions}

In conclusion, jointly modelling longitudinal parasite count and time-to-new clinical malaria improved precision in log hazard ratio estimates for clinical malaria when compared with the conventional $\mathrm{Cox} \mathrm{PH}$ model with time-dependent parasite count. The improved precision of joint modelling may improve study efficiency and allow for design of clinical trials with relatively lower sample sizes with increased power.

\section{Competing interests}

The authors declare that they have no competing interests.

\section{Acknowledgements and Funding}

Research reported in this publication was supported by the Fogarty International Center of the National Institutes of Health under Award Number D43TW010075. The work was also partially supported through the DELTAS Sub-Saharan Africa Consortium for Advanced Biostatistics (SSACAB) programme and the UK government with funding from the Wellcome Trust Award Number 107754/Z/15/Z.

\section{Authors' contributions}

\begin{tabular}{|l|c|c|c|c|c|c|c|c|}
\hline Authors' contributions & CCS & LNK & AGB & MM & DPM & MGH & MKL & TFC \\
\hline Research concept and design & $\checkmark$ & $\checkmark$ & -- & -- & $\checkmark$ & -- & $\checkmark$ & $\checkmark$ \\
\hline Collection and/or assembly of data & -- & -- & $\checkmark$ & -- & $\checkmark$ & -- & $\checkmark$ & -- \\
\hline Data analysis and interpretation & $\checkmark$ & $\checkmark$ & $\checkmark$ & $\checkmark$ & $\checkmark$ & $\checkmark$ & $\checkmark$ & $\checkmark$ \\
\hline Writing the article & $\checkmark$ & $\checkmark$ & $\checkmark$ & $\checkmark$ & $\checkmark$ & $\checkmark$ & $\checkmark$ & $\checkmark$ \\
\hline Critical revision of the article & $\checkmark$ & $\checkmark$ & $\checkmark$ & $\checkmark$ & $\checkmark$ & $\checkmark$ & $\checkmark$ & $\checkmark$ \\
\hline Final approval of article & $\checkmark$ & $\checkmark$ & $\checkmark$ & $\checkmark$ & $\checkmark$ & $\checkmark$ & $\checkmark$ & $\checkmark$ \\
\hline Statistical analysis & $\checkmark$ & $\checkmark$ & -- & $\checkmark$ & -- & $\checkmark$ & -- & $\checkmark$ \\
\hline
\end{tabular}

\section{Publication history}

EIC: Jimmy Efird, East Carolina University, USA.

Received: 04-Mar-2019 Final Revised: 17-Apr-2019

Accepted: 20-Apr-2019 Published: 05-Jun-2019

\section{References}

1. World Health Organisation (WHO). World Malaria Report 2017. 2017; 186. | Article

2. Barber BE, William T, Grigg MJ, Menon J, Auburn S, Marfurt J, Anstey NM and Yeo TW. A prospective comparative study of knowlesi, falciparum, and vivax malaria in Sabah, Malaysia: high proportion with severe disease from Plasmodium knowlesi and Plasmodium vivax but no mortality with early referral and artesunate therapy. Clin Infect Dis. 2013; 56:383-97. | Article I PubMed

3. Kasirye R, Grosskurth H, Munderi P, Levin J, Anywaine Z, Nunn A, Kamali $A$ and Baisley K. Longitudinal effect of CD4 by cotrimoxazole use on malaria incidence among HIV-infected Ugandan adults on antiretroviral therapy: a randomized controlled study. Malar J. 2016; 15:361. | Article | PubMed Abstract | PubMed FullText

4. Loucoubar C, Grange L, Paul R, Huret A, Tall A, Telle O, Roussilhon C, Faye J, Diene-Sarr F, Trape JF, Mercereau-Puijalon O, Sakuntabhai A and Bureau JF. High number of previous Plasmodium falciparum clinical episodes increases risk of future episodes in a sub-group of individuals. PLoS One. 2013; 8:e55666. | Article | PubMed Abstract | PubMed FullText

5. Guo X and Carlin BP. Separate and Joint Modeling of Longitudinal and Event Time Data Using Standard Computer Packages. Am Stat. 2004; 58:16-24.

6. White NJ. Malaria parasite clearance. Malar J. 2017; 16:88. | Article | PubMed Abstract | PubMed FullText

7. Kalbfleisch JD and Prentice RL. The Statistical Analysis of Failure Time Data. New York: John Wiley and Sons. 2002.
8. Song $X$, Davidian $M$ and Tsiatis AA. A semiparametric likelihood approach to joint modeling of longitudinal and time-to-event data. Biometrics. 2002; 58:742-53.. | Article | PubMed

9. Neuenschwander B and Branson M. Modeling missingness for timeto-event data: a case study in osteoporosis. J Biopharm Stat. 2004; 14:1005-19. | Article | PubMed

10. Taylor JM and Wang Y. Surrogate markers and joint models for longitudinal and survival data. Control Clin Trials. 2002; 23:626-34. | Article I PubMed

11. Ibrahim JG, Chen M-H and Sinha D. Bayesian methods for joint modeling of longitudinal and survival data with applications to cancer vaccine trials. Stat Sin. 2004; 14:863-884.

12. Chen Q, May RC, Ibrahim JG, Chu H and Cole SR. Joint modeling of longitudinal and survival data with missing and left-censored timevarying covariates. Stat Med. 2014; 33:4560-76. | Article I PubMed Abstract | PubMed FullText

13. Chi YY and Ibrahim JG. Joint models for multivariate longitudinal and multivariate survival data. Biometrics. 2006; 62:432-45. | Article | PubMed

14. Brown ER and Ibrahim JG. A Bayesian semiparametric joint hierarchical model for longitudinal and survival data. Biometrics. 2003; 59:221-8. I Article | PubMed

15. Buchwald AG, Sixpence A, Chimenya M, Damson M, Sorkin JD, Wilson ML, Seydel K, Hochman S, Mathanga DP, Taylor TE and Laufer MK. Clinical Implications of Asymptomatic Plasmodium falciparum Infections in Malawi. Clin Infect Dis. 2019; 68:106-112. | Article | PubMed 
Stanley et al., Journal of Medical Statistics and Informatics 2019, http://www.hoajonline.com/journals/pdf/2053-7662-7-1.pdf

16. World Health Organization (WHO). Epidemiological profile of malaria in Malawi in 2015. 2017. I Pdf

17. Bennett A, Kazembe L, Mathanga DP, Kinyoki D, Ali D, Snow RW and Noor AM. Mapping malaria transmission intensity in Malawi, 2000-2010. Am J Trop Med Hyg. 2013; 89:840-849. | Article | PubMed Abstract | PubMed FullText

18. Walldorf JA, Cohee LM, Coalson JE, Bauleni A, Nkanaunena K, KapitoTembo A, Seydel KB, Ali D, Mathanga D, Taylor TE, Valim C and Laufer MK. School-Age Children Are a Reservoir of Malaria Infection in Malawi. PLoS One. 2015; 10:e0134061. | Article | PubMed Abstract | PubMed FullText

19. Ibrahim JG, Chen MH and Sinha D. Bayesian Survival Analysis. New York: Springer. 2001.

20. StataCorp. Stata Statistical Software: Release. 2017; 15.

21. R Core Team. A language and environment for statistical computing. $\mathbf{R}$ Found Stat Comput Vienna, Austria. 2017; R Foundation for Statistical Computing. 2017.

22. Spiegelhalter DJ, Best NG, Carlin BP and Van Der Linde A. Bayesian measures of model complexity and fit. J R Stat Soc Ser B Stat Methodol. 2002; 64:583-616. | $\underline{\mathrm{Pdf}}$

23. Ibrahim JG, Chu $\mathrm{H}$ and Chen LM. Basic concepts and methods for joint models of longitudinal and survival data. J Clin Oncol. 2010; 28:2796801. | Article | PubMed Abstract | PubMed FullText

24. Lawrence Gould A, Boye ME, Crowther MJ, Ibrahim JG, Quartey G, Micallef $S$ and Bois FY. Joint modeling of survival and longitudinal nonsurvival data: current methods and issues. Report of the DIA Bayesian joint modeling working group. Stat Med. 2015; 34:2181-95. | Article | PubMed Abstract | PubMed FullText

25. Mathanga DP, Tembo AK, Mzilahowa T, Bauleni A, Mtimaukenena K, Taylor TE, Valim C, Walker ED and Wilson ML. Patterns and determinants of malaria risk in urban and peri-urban areas of Blantyre, Malawi. Malar J. 2016; 15:590. | Article | PubMed Abstract | PubMed FullText

26. Alemu A, Tsegaye W, Golassa $L$ and Abebe G. Urban malaria and associated risk factors in Jimma town, south-west Ethiopia. Malar J. 2011; 10:173. | Article | PubMed Abstract | PubMed FullText

27. Haque $U$, Glass GE and Bomblies A. Risk factors associated with multiple malaria infections in Bangladesh. Am J Trop Med Hyg. 2012; 87:403. | Pdf

28. Haque U, Glass GE, Bomblies A, Hashizume M, Mitra D, Noman N, Haque W, Kabir MM, Yamamoto T and Overgaard HJ. Risk factors associated with clinical malaria episodes in Bangladesh: a longitudinal study. Am J Trop Med Hyg. 2013; 88:727-732. | Article | PubMed Abstract | PubMed FullText

29. Biswas S, Seth RK, Tyagi PK, Sharma SK and Dash AP. Naturally acquired immunity and reduced susceptibility to falciparum malaria in two subpopulations of endemic eastern India. Scand J Immunol. 2008; 67:177-84. | Article | PubMed

\section{Citation:}

Stanley CC, Kazembe LN, Buchwald AG, Mukaka M, Mathanga DP, Hudgens MG, Laufer MK and Chirwa TF. Joint modelling of time-to-clinical malaria and parasite count in a cohort in an endemic area. J Med Stat Inform. 2019; 7:1.

http://dx.doi.org/10.7243/2053-7662-7-1 